\title{
Design and realization visible light communication transmitter circuit
}

\author{
Shuai Liu, Gang Cheng, Yuming Zhu, Xiao Wang \\ China Satellite Maritime Tracking and Control Department, Jiangyin, 214431, China
}

\begin{abstract}
In this paper, the front-end transmit visible light communication circuit design are discussed. The first part focuses on the design of visible light emission circuit in response to the high speed, low power consumption characteristics of the LED light source to do a consideration, based on the detection of light intensity, respectively, the use of transistors and amplifiers transmitting circuit three programs; The second section focuses on the three circuit performance tests and the results were analyzed.
\end{abstract}

Keywords: Visible light communication; LED; photodiode; Amplifier.

\section{Introduction}

VLC as an emerging communications technology is a combination of optical communication and wireless communication technology between the two is an effective complement contemporary means of communication. The basic principle of visible light communication is the use of LED emitted by human eye can not detect the high-speed bright flashing signal to carry digital information. As the basis for the visible light communication circuit implementation, the focus of this study is designed to emit visible light communications front-end circuits, digital signal into an optical signal, and a short distance high-speed transmission.

\section{Visible light communication transmitter circuit design analysis of key issues}

In theory, most of the current modulation scheme already in use is suitable for visible light communication system, but for now, all developed an optical communication system or the use of mostly light-intensity modulation direct detection (IM-DD), the use of the modulation signal off keying (OOK).

Since the LED forward voltage characteristics is very steep (ie forward dynamic resistance very small), so the power supply to the LED is not like an ordinary incandescent lamp as a direct voltage source power supply, voltage fluctuation or the slightly increases, the current increases abruptly, so that the LED will burn. Therefore, in order to stabilize the LED current, ensure LED work normally and stably, we need to build a LED driver circuit. Meanwhile, LED visible light communication, since a single bulb lighting range is limited, more general application is several light bulbs strung together, which requires the driver circuit can provide enough current for the lamp to ensure LED to provide the necessary brightness. Moreover, since the circuit need to provide up to hundreds of megabytes of data transfer rate is also necessary to consider the response time of the LED, the LED drive circuit which also put forward higher requirements.

Thus, for modulating white LED driving circuit general requirements are: First, the stability of the output optical power as large as possible. Optical power output, the longer the signal light can be transmitted from the system wide coverage. Second, the bandwidth of the circuit to be able to cover the spectral range transmission of electrical signals, and the signal having the smallest possible nonlinear distortion characteristics.

\section{Implementations of the transmitting circuit}

Taking into account the paper will use the on-off keying (OOK) modulated signal for visible light communication transmitter circuit design, the following three options: 
Option One: Since LEDs lamps at the same time to communicate, to complete the task itself is also illuminated, so, for modulating light LEDs can be placed directly on the power line. By controlling the on-off power lines, can achieve high-speed LEDs lights blinking. This circuit is shown in Figure 1.

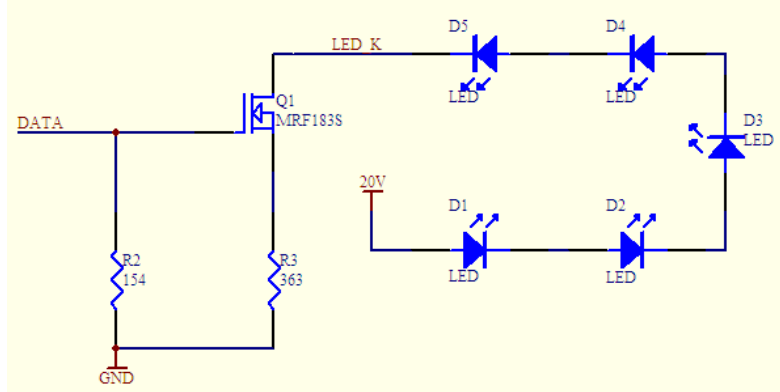

Figure 1. Transmitting FET circuit composed of tubes

Figure 1 transistor used to enhanced N-channel FET, model MRF183S. MOTOROLA MRF183S is a designed for the high-gain, broadband applications designed for RF power FET, 28V voltage above its bandwidth up to $1 \mathrm{GHz}$, the conversion efficiency of $33 \%$, can provide up to $45 \mathrm{~W}$ of power output.

Option Two: In addition to considering the use of transistors, you can also use an amplifier in the form of a control circuit. Circuit still choose to install the power line, the difference is that the switch to control two-stage amplifier circuit structure to provide a bias and a load signal for the LED lamps. The last part of the second-stage amplifier circuit, Figure 2 is an enlarged section showing the circuit.

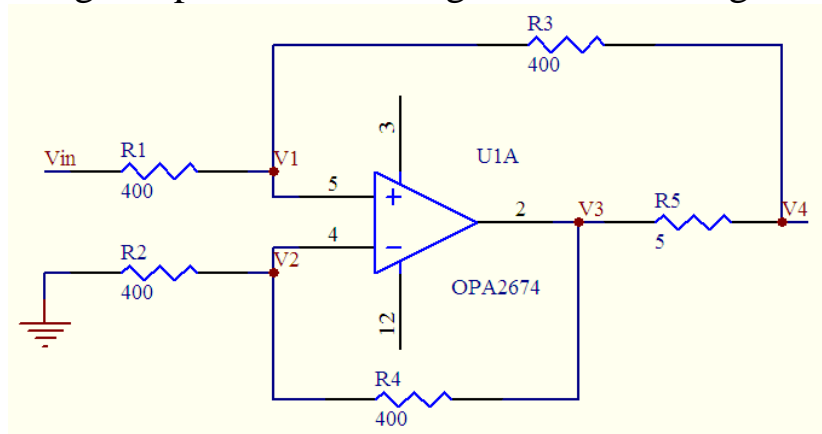

Figure 2. The second stage amplifier circuit of option II

The circuit is intended for use in an amplifier chip model OPA2674, the same series of OPA2670, OPA2673 is also one of the circuit chips for use under consideration, but because of the two chip packaging is RGV QFN-16, and the multi-pin small in volume, easy manual welding, so the choice for the OPA2674 chip package of sO-14. The TI chip is produced by a wideband, high output current, high linearity, and with a limited flow protection dual op-amp chip, mainly as a differential amplifier. This chip is a current feedback amplifier, with respect to voltage feedback amplifier biggest advantage is its AC performance (including bandwidth and distortion) is relatively independent of voltage gain. This means that, as long as appropriate adjustments to external resistor with, especially the feedback resistor, the circuit can keep the original performance in achieving good band gain changes at the same time. If you work in a single $+12 \mathrm{~V}$ power state that the chip consumes only low $9 \mathrm{~mA} / \mathrm{ch}$ can generate a quiescent current of up to $500 \mathrm{~mA}$ of output. Anti-noise performance of the chip is also very well compared to other current feedback amplifier, this chip between the voltage and current noise do a good balance.

Option Three: The program on the basis of the second scheme, a number of changes to the circuit, the original dual power supply design into a single $+12 \mathrm{~V}$ power supply. The specific design of the circuit is shown in Figure 3. Similarly, in order to LED lamps provide sufficient bias, under a single power supply circuit in the state, the second-stage circuit has been modified, the analysis part of the circuit alone. According to analysis before the amplifier output voltage size:

$V_{3}=V_{2}-\left[\left(5-V_{2}\right) / 390\right] \times 390=2 V_{2}-5=2 V_{1}-5$

Figure $4, V_{4}$ is at voltage size: 
$V_{4}=V_{1}-\left[\left(V_{\text {in }}-V_{1}\right) / 390\right] \times 390=2 V_{1}-V_{\text {in }}$

$2.5 \mathrm{ohm}$ resistor flowing current size:

$I_{2}=\left[\left(2 V_{1}-5\right)-\left(2 V_{1}-V_{\text {in }}\right)\right] / 2.5=\left(V_{\text {in }}-5\right) / 2.5$

It is aiming to provide $300 \mathrm{~mA}$ DC bias requires pre-divider circuit.

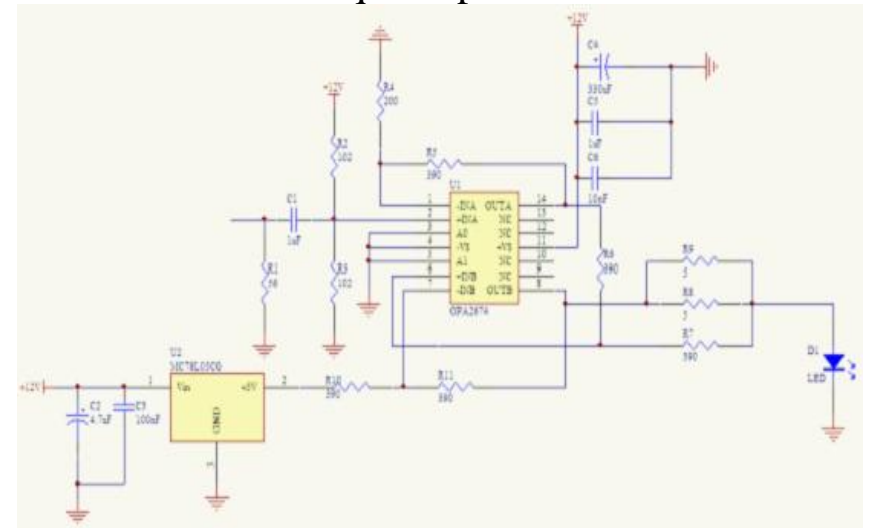

Figure 3. Single power amplifier transmitting circuit state

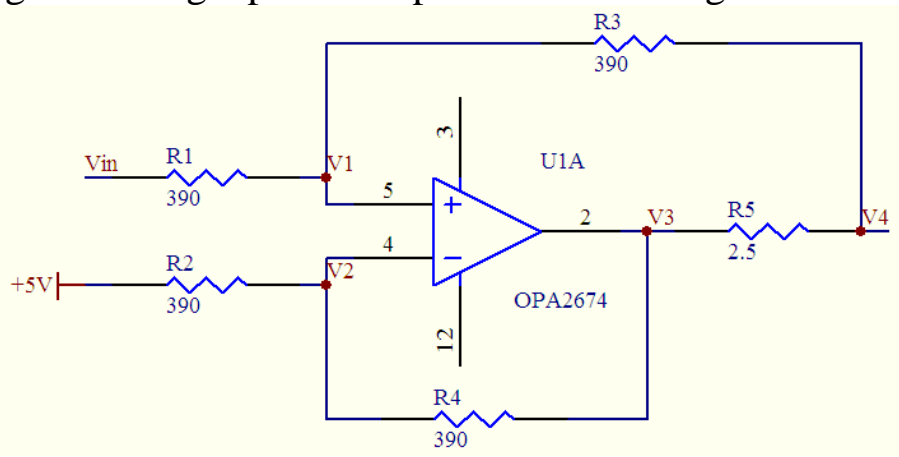

Figure 4. The program of the second amplifier stage III

\section{System Performance Testing and Analysis}

\subsection{Test equipment used}

Multimeters, signal sources, oscilloscopes DS1204B, DC voltage source.

\subsection{Transmitting circuit performance test}

As shown in Figure 5, respectively, to the transmitting end circuitry, digital signal source to generate a sine wave of the desired frequency, the signal voltage value at the output of the test lamp beads and observing the degree of fidelity of the output signal. Specific test results are shown in Figure 6-7. Among them, the program two and three similar program performance, it is not listed separately.

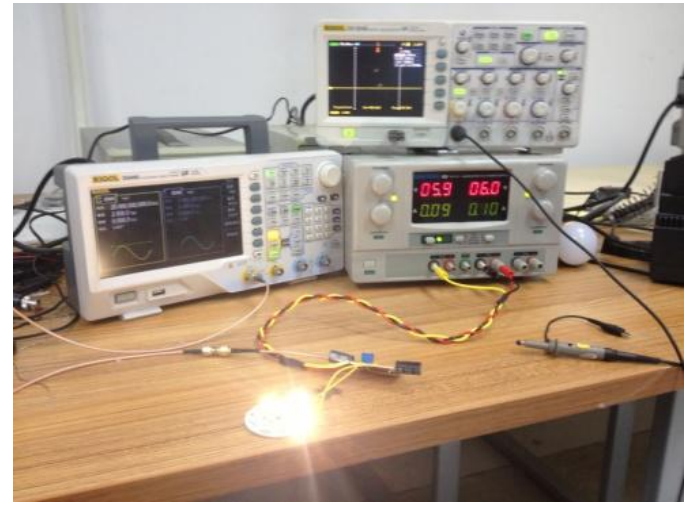

Figure 5. The transmission side circuit debugging environment

\subsection{Analysis of test results}

The transmission side circuit, the three programs can achieve the basic signal modulation rate of the order of megabytes. The transmitting circuit op amp composed of many problems. It was found 
that, for the input sine wave, an op amp can be properly amplified, but the second stage op amp inverting terminal waveform distortion has appeared, and with the increase of frequency distortion becomes severe. After analysis, the reasons are: first, the impact of the experimental equipment circuits. Oscilloscope test leads itself capacitance effect, when the pen access the op amp inverting terminal when the capacitance effects lead affects the normal performance of the circuit, resulting in distortion of the output signal oscilloscope. Second, the circuit layout itself affects the op amp performance of the play. Circuit layout is such that high-frequency signals on the line influence each other, eventually leading to the distortion of the output signal.

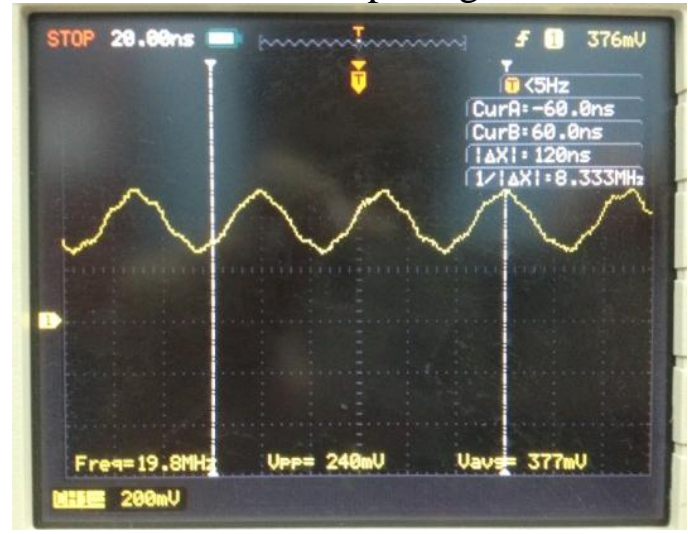

Figure 6. Option One, an input frequency of $20 \mathrm{MHz}$ output waveform when a sine wave.

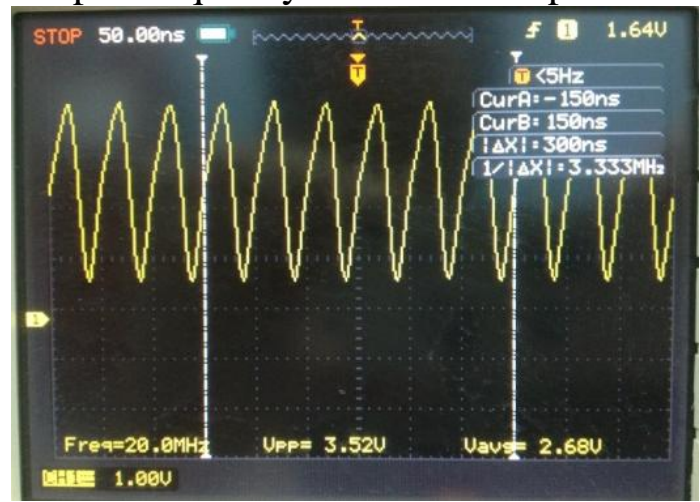

Figure 7. Option II transmission side input frequency of $20 \mathrm{MHz}$ output waveform when a sine wave.

\section{Summary}

Object thesis is currently the more popular indoor LED wireless visible light communication technology, the use of theoretical analysis and hardware implementation of the method, the subject of a front-end circuit provides several implementations of the technology.

Design discussed in the visible light emission circuit, based on the characteristics of white LED light source, the use of light-intensity modulation direct detection technique, the signal is a simple OOK modulation, and gives three different implementations.

\section{References}

[1]. Wang Xiaoming. Indoor LED visible physical layer of wireless communication systems research [D]. Jiangsu University Master Thesis 2008:1-3

[2]. Yun Wenyue. Laboratory Study modulation and demodulation technology visible light communication system of Changchun University of Technology master's degree thesis 2008

[3]. Zhang Jiankun, Yang Yu. Analysis indoor visible light communication modulation method. Section 4. 2011. China Laser

[4]. Hoa Le Minh, Dominic O’Brien, Grahame Faulkner, Lubin Zeng, Kyungwoo Lee, Daekwang Jung, YunJe Oh, and Eun Tae Won."100-Mb/s NRZ Visible Light Communications Using a Postequalized White LED”.IEEE PHOTONICS TECHNOLOGY LETTERS.2009 